\title{
Conformal symmetry algebra of the quark potential and degeneracies in the hadron spectra
}

\author{
M. Kirchbach \\ Instituto de Física, Universidad Autónoma de San Luis Potosí, \\ Av. Manuel Nava 6, San Luis Potosí, S.L.P. 78290, México
}

\begin{abstract}
The essence of the potential algebra concept [Y. Alhassid, F. Gürsey, F. Yachello. Phys. Rev. Lett. 50 (1983)] is that quantum mechanical free motions of scalar particles on curved surfaces of given isometry algebras can be mapped on 1D Schrödinger equations with particular potentials. As long as the Laplace-Beltrami operator on a curved surface is proportional to one of the Casimir invariants of the isometry algebra, free motion on the surface is described by means of the eigenvalue problem of that very Casimir operator. In effect, the excitation modes considered are classified according to the irreducible representations of the algebra of interest and are characterized by typical degeneracies. In consequence, also the spectra of the equivalent Schrödinger operators are classified according to the same irreducible representations and carry the same typical degeneracies. A subtle point concerns the representation of the algebra elements which may or may not be unitarily equivalent to the standard one generating classical groups like $S O(n), S O(p, q)$, etc. To be specific, any similarity transformations of an algebra that underlies, say, an orthogonal group, always conserve the commutators among the elements, but a non-unitarily transformed algebra must not generate same group. One can then consider the parameters of the non-unitary similarity transformation as group symmetry breaking scales and seek to identify them with physical observables. We here use the potential algebra concept as a guidance in the search for an interaction describing conformal degeneracies. For this purpose we subject the $s o(4) \subset s o(2,4)$ isometry algebra of the $S^{3}$ ball to a particular non-unitary similarity transformation and obtain a deformed isometry copy to $S^{3}$ such that free motion on the copy is equivalent to a cotangent perturbed motion on $S^{3}$, and to the 1D Schrödinger operator with the trigonometric Rosen-Morse potential as well. The latter presents itself especially well suited for quark-system studies insofar as its Taylor series decomposition begins with a Cornell-type potential and in accord with lattice QCD predictions. We fit the strength of the cotangent potential to the spectra of the unflavored high-lying mesons and obtain a value compatible with the light dilaton mass. We conclude that while the conformal group symmetry of QCD following from $A d S_{5} / C F T_{4}$ may be broken by the dilaton mass, it still may be preserved as a symmetry algebra of the potential, thus explaining the observed conformal degeneracies in the unflavored hadron spectra, both baryons and mesons.
\end{abstract}

PACS numbers: $12.39 . \mathrm{Jh}, 24.85 .+\mathrm{p}$

Explorer, your footsteps are the trail, and nothing else: explorer, no trail to follow, advancing trails are blazed. after Antonio Machado

To the 70th birthday of Francesco Yachello

\section{THE POTENTIAL ALGEBRA CONCEPT}

Group theoretical approaches to both bound and scattering state problems in physics have played a pivotal róle in our understanding of spectra classifications. It is a well known fact, that several of the exactly solvable quantum mechanical potentials give rise to spectra which fall into the irreducible representations of certain Lie group algebras. As a representative case, we wish to mention the widely studied class of Natanzon potentials [1] known to produce spectra that populate multiplets of the pseudo-rotational algebras $\mathrm{so}(2,2) / \mathrm{so}(2,1)$ 2]. This phenomenon has been well understood in casting the Hamiltonians with the potentials under consideration as Casimir invariants of so $(2,2) / \operatorname{so}(2,1)$ algebras in representations not necessarily unitarily equivalent to the pseudo-rotational and referred to as "potential algebras" [3]- [4]. The hyperbolic Pöschl-Teller-, Scarf-, and Eckart potentials stand for three popular interactions having su(1,1) symmetry algebras. 


\section{A. The so $(2,1)$ isometry algebra of the one-sheeted hyperbolic plane and the $\frac{-\lambda(\lambda+1)}{\cosh ^{2} \rho}$ potential}

The essence of the potential algebra concept pioneered in [3] is that quantum mechanical free motions on curved surfaces of given isometry algebras can be transformed, upon an appropriate change of variables, into 1D Schrödinger equations with particular potentials. As long as the Laplace-Beltrami operator on a curved surface is proportional to one of the Casimir invariants of the associated isometry algebra, free motion on the surface is described by means of the eigenvalue problem of that very Casimir invariant. In consequence, the spectrum of the related potentials will be classified according to the irreducible representations of the algebras under consideration and will be characterized by typical degeneracies. In particular, in [3] attention has been drawn to the fact that the bound and scattering states of the hyperbolic $V=-\lambda(\lambda+1) / \cosh ^{2} \rho$ potential (it can be part of either the Pöschl-Teller-, or the Scarf interactions), fall into irreducible representations of the $s u(1,1) \sim s o(2,1)$ algebra because this potential can be embedded into the Casimir invariant, $\mathcal{C}$, of the pseudo-rotational algebra, $s u(1,1) \sim s o(2,1)$, acting as an isometry algebra of the one-sheeted hyperboloid, $x^{2}+y^{2}-z^{2}=1^{2}$, an $A d S_{2}$ space. The algebra is spanned by the generators

$$
\left[J_{z}, J_{ \pm}\right]= \pm J_{ \pm}, \quad\left[J_{+}, J_{-}\right]=-2 J_{z}, \quad \mathcal{C}=J_{z}^{2}+J_{z}-J_{-} J_{+}=-\Delta
$$

with $\Delta$ being the Laplacian on the one sheeted-hyperboloid [5]. Upon a parametrization of the above surface as

$$
x=\cosh \rho \cos \varphi, \quad y=\cosh \rho \sin \varphi, \quad z=\sinh \rho, \quad \mathcal{C}=\frac{1}{\cosh \rho} \frac{\partial}{\partial \rho} \cosh \rho \frac{\partial}{\partial \rho}+\frac{m^{2}}{\cosh ^{2} \rho},
$$

the quantum mechanical free motion of a scalar particle of mass $\mu$ on it is described by the $\left(-\frac{\hbar^{2}}{2 \mu}\right) \mathcal{C}$ eigenvalue problem as,

$$
-\frac{\hbar^{2}}{2 \mu} \mathcal{C} \mathcal{Z}_{k}^{m}(\rho, \varphi)=-\frac{\hbar^{2}}{2 \mu} k(k-1) \mathcal{Z}_{k}^{m}(\rho, \varphi), \quad \frac{\partial}{\partial \varphi} \mathcal{Z}_{k}^{m}(\rho, \varphi)=m \mathcal{Z}_{k}^{m}(\rho, \varphi),
$$

where $\mathcal{Z}_{k}^{m}(\rho, \varphi)$ are the $s o(2,1)$ representation functions, while $k$, and $m$ stand for the corresponding representation labels. In the following, we chose to work in the dimensionless units, $\hbar=1,2 \mu=1$. In changing now variables to $\mathcal{Z}_{k}^{m}(\rho, \varphi)=\frac{U_{k}^{m}(\rho, \varphi)}{\sqrt{\cosh \rho}}$, converts (3) to the $1 D$ Schrödinger equation with the $\frac{-\lambda(\lambda+1)}{\cosh ^{2} \rho}$ potential,

$$
\begin{aligned}
& \mathcal{H} U_{k}^{m}(\rho, \varphi)=\epsilon_{k} U_{k}^{m}(\rho, \varphi), \quad \mathcal{H}=-\frac{\partial^{2}}{\partial \rho^{2}}-\frac{m^{2}-\frac{1}{4}}{\cosh ^{2} \rho}+\frac{1}{4}, \quad \lambda=|m|-\frac{1}{2}, \\
& \epsilon_{k}=-\left(|m|-\frac{1}{2}-n\right)^{2}+\frac{1}{4}=-k(k-1), \quad k=|m|-n .
\end{aligned}
$$

The $k$ label can be fixed to a positive integer which would mean choosing an infinite unitary discrete series representation of $s o(1,2)$ with $|m|=k+n$, and $n=0,1,2, \ldots$ Stated differently, the states bound within hyperbolic potentials of the type $\left[-(|m|+1 / 2)(|m|-1 / 2) / \cosh ^{2} \eta\right]$ with $|m|=k, k+1, \ldots$ will populate discrete unitary irreducible representations of $s o(2,1)$. This is explained by the circumstance that the associated Schrödinger Hamiltonian allows for a map on a linear function of the $s u(1,1) \sim s o(2,1)$ Casimir operator according to,

$$
\frac{1}{\sqrt{\cosh \rho}} \mathcal{H} U_{k}^{m}(\rho, \varphi)=-\left(\mathcal{C}+\frac{1}{4}\right) \frac{U_{k}^{m}(\rho, \varphi)}{\sqrt{\cosh \rho}}=-\left[k(k-1)+\frac{1}{4}\right] \frac{U_{k}^{m}(\rho, \varphi)}{\sqrt{\cosh \rho}} .
$$

One says, that the hyperbolic $\frac{-\lambda(\lambda+1)}{\cosh ^{2} \rho}$ potential has $s u(1,1) \sim s o(2,1)$ symmetry algebra, or, equivalently, that the isometry algebra $s u(1,1) \sim s o(2,1)$ of the one-sheeted hyperboloid is the potential algebra of the $\frac{-\lambda(\lambda+1)}{\cosh ^{2} \rho}$ interaction for the particular $\lambda=\left(|m|-\frac{1}{2}\right)$ values. The scattering states would be classified according to continuous-series representations of $s u(1,1)$.

\section{B. The so( 1,2$)$ isometry algebra of the two-sheeted hyperbolic plane and the Eckart potential}

In a similar way, the hyperbolic $\frac{\lambda(\lambda-1)}{\sinh ^{2} \eta}$ potential can be related to the Casimir invariant of the isometry algebra of the two-sheeted hyperboloid, $z^{2}-x^{2}-y^{2}=1$, which is $s o(1,2)$, and can be parametrized as [5]

$$
x=\sinh \eta \cos \varphi, \quad y=\sinh \eta \sin \varphi, \quad z=\cosh \eta, \quad \mathcal{C}=\frac{1}{\sinh \eta} \frac{\partial}{\partial \eta} \sinh \eta \frac{\partial}{\partial \eta}-\frac{m^{2}}{\sinh ^{2} \eta} .
$$


Also in this case, the quantum mechanical free motion is described in terms of the eigenvalue problem of the relevant Casimir operator, $\mathcal{C}$,

$$
-\frac{\hbar^{2}}{2 \mu} \mathcal{C} Y_{k}^{m}(\eta, \varphi)=-\frac{\hbar^{2}}{2 \mu} k(k+1) Y_{k}^{m}(\eta, \varphi), \quad Y_{k}^{m}(\eta, \varphi)=P_{k}^{|m|}(\cosh \eta) e^{i m \varphi},
$$

where $Y_{k}^{m}(\eta, \varphi)$ are the pseudo-spherical harmonics. Also here, a suited variable change,

$$
Y_{k}^{m}(\eta, \varphi)=\frac{U_{k}^{m}(\eta, \varphi)}{\sqrt{\sinh \eta}}
$$

converts the free motion on the upper sheet, $\mathbf{H}_{+}^{2}$, of the hyperbolic plane, into an 1D Schrödinger equation, this time with the Eckart potential [ 6 ] according to

$$
\begin{array}{r}
\mathcal{H} U_{k}^{m}(\eta, \varphi)=\epsilon_{k} U_{k}^{m}(\eta, \varphi), \quad \mathcal{H}=-\frac{\partial^{2}}{\partial \eta^{2}}+\frac{m^{2}-\frac{1}{4}}{\sinh ^{2} \eta}+\frac{1}{4}, \quad \lambda=|m|+\frac{1}{2}, \\
\epsilon_{k}=-\left(|m|+\frac{1}{2}+n\right)^{2}+\frac{1}{4}=-k(k+1), \quad k=|m|+n, \quad n, k=0,1,2, \ldots, \quad|m| \in[0, k] .
\end{array}
$$

Also in this case, the conclusion can be drawn that the isometry algebra of the surface on which the free motion takes place, acts as a symmetry algebra of the potential appearing in the equivalent Schrödinger equation. However, a curious and most interesting situation occurs upon perturbing the aforementioned free motion by a coth $\eta$ interaction, which results in [2]

$$
-\frac{\hbar^{2}}{2 M}(\mathcal{C}+2 b \operatorname{coth} \eta) F_{t}^{\tilde{m}}(\eta, \varphi)=-\frac{\hbar^{2}}{2 M} \epsilon_{t} F_{t}^{\widetilde{m}}(\eta, \varphi), \quad \epsilon_{t}=t(t+1)+\frac{b^{2}}{\left(t+\frac{1}{2}\right)^{2}}, \quad t=n+|\widetilde{m}| .
$$

Despite non-commutativity of the perturbance with $\mathcal{C}$, the wave functions, $F_{t}^{\widetilde{m}}(\eta, \varphi)$, in (10) continue transforming according to the finite-dimensional non-unitary representations of the $s o(1,2)$ algebra, and the corresponding excitations are characterized by same degeneracies, as the kinetic motion. This becomes apparent upon changing variables in (10) according to (8), in which case one arrives at the well known 1D Schrödinger equation with the Eckart potential. In [7] this peculiarity of the Eckart potential has been explained by showing that the Hamiltonians of the $\operatorname{coth} \eta$-perturbed-, and the free motions on $\mathbf{H}_{+}^{2}$ are related by a non-unitary similarity transformation, that can be directly read off from the decomposition of the perturbed solutions in the basis of the free ones. In dimensionless units one finds,

$$
\begin{aligned}
(\mathcal{C}+2 b \operatorname{coth} \eta) \mathbf{X}_{t}(\eta, \varphi) & =\left[\widetilde{\mathcal{C}}+\frac{\alpha_{t}^{2}}{4}\right] \mathbf{X}_{t}(\eta, \varphi), \quad \widetilde{\mathcal{C}}=e^{-\frac{\alpha_{t} \eta}{2}} A_{t}(\varphi) \mathcal{C} e^{\frac{\alpha_{t} \eta}{2}} A_{t}^{-1}(\varphi), \quad \alpha_{t}=\frac{2 b}{t+\frac{1}{2}} \\
\mathbf{X}_{t}(\eta, \varphi) & =e^{-\frac{\alpha_{t} \eta}{2}}\left(\begin{array}{c}
F_{t}^{0}(\eta) e^{i 0 \varphi} \\
F_{t}^{1}(\eta) e^{i \varphi} \\
\ldots \\
F_{t}^{t}(\eta) e^{i l \varphi}
\end{array}\right)=A_{t}(\varphi) e^{-\frac{\alpha_{t} \eta}{2}}\left(\begin{array}{c}
Y_{t}^{0}(\eta, \varphi) \\
Y_{t}^{1}(\eta, \varphi) \\
\ldots \\
Y_{t}^{t}(\eta, \varphi)
\end{array}\right) .
\end{aligned}
$$

Here, $A_{t}(\varphi)$ are matrices,

$$
A_{t}(\varphi)=\left(\begin{array}{cccc}
a_{11}^{t} & a_{12}^{t} e^{-i \varphi} & \ldots & a_{1(t+1)}^{t} e^{-i t \varphi} \\
0 & a_{22}^{t} & \ldots & a_{2(t+1)}^{t} e^{-i(t-1) \varphi} \\
\ldots & \ldots & \ldots & \ldots \\
0 & 0 & 0 & a_{(t+1)(t+1)}^{t}
\end{array}\right)
$$

which determine the decomposition of the representation functions, $\mathbf{X}_{t}(\eta, \varphi)$, of the similarity transformed Casimir operator, $\widetilde{\mathcal{C}}$, into the basis of the pseudo-spherical harmonics, the representation functions of the algebra underlying the pseudo-orthogonal group $S O(1,2)$. As long as the $t$ label takes same values as $k$ in (9), and $|\widetilde{m}|$ obeys same branching rule with respect to $t$ as $|m|$ to $k$, the $(2 t+1)$-fold degeneracies of the full Eckart potential are indistinguishable from the $(2 k+1)$-fold ones of the free motion on $\mathbf{H}_{+}^{2}$. Important, the level splittings will be modified through the presence of the representation constant $\alpha_{t}$ in (11). Such type of differences between pseudo-rotational spectra reveal changes in the representation of the algebra. Explicit expressions for the $A_{t}(\varphi)$ matrices can be found in [7]. It is also important to be aware of the fact that eq. (111) is not valid at the operator level, i.e. $(\mathcal{C}+2 b \operatorname{coth} \eta) \neq\left(\widetilde{\mathcal{C}}+\frac{\alpha_{t}^{2}}{4}\right)$. Its 

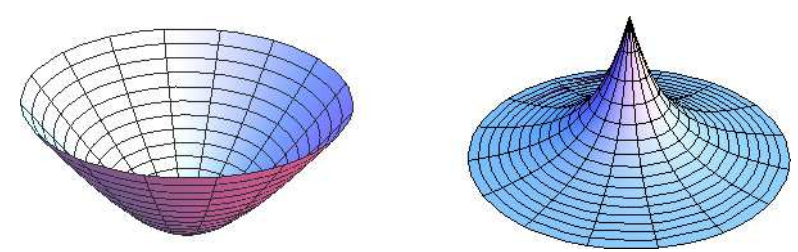

FIG. 1: The $\operatorname{coth} \eta$ perturbed motion on $\mathbf{H}_{+}^{2}$ (left) is equivalent to free motion on the deformed surface (right).Obviously, the figure on the right is not a symmetric space of the $S O(1,2)$ group. We conclude that though the Eckart potential has $s o(1,2)$ as a symmetry algebra, the $S O(1,2)$ group symmetry of the free motion has been broken by the scale introduced by the potential strength, causing a deformation of the $\mathbf{H}_{+}^{2}$ metric.

validity at the level of the eigenvalue problems is guaranteed by virtue of certain class of recurrence relations among associated Legendre functions, listed in [7]. The non-unitary similarity transformation in (11), (12) is distinct from the one considered in [8] whose main purpose has been to prove that the $\operatorname{coth} \eta$-perturbed motion on $\mathbf{H}_{+}^{2}$ gives rise to a Natanzon class potential. Notice that the $\operatorname{coth} \eta$-perturbed motion on $\mathbf{H}_{+}^{2}$ is equivalent to free motion on the $\exp (-2 b \eta)$ rescaled hyperbolic space, the lowest solution of (10), visualized in Fig. 1.

\section{The so(3) isometry algebra of $S^{2}$ and the cotangent perturbed rigid rotator}

As long as $s o(1,2)$, and $s o(3)$ are related by a Wigner rotation, $\mathcal{C}$ in eq. (6) and standard squared orbital angular momentum $\mathbf{L}^{2}$,

$$
\mathbf{L}^{2}=-\frac{1}{\sin \theta} \frac{\partial}{\partial \theta} \sin \theta \frac{\partial}{\partial \theta}-\frac{1}{\sin ^{2} \theta} \frac{\partial^{2}}{\partial \varphi^{2}},
$$

are related by a complexification of the polar angle,

$$
\mathbf{L}^{2} \stackrel{\theta \rightarrow i \eta}{\longrightarrow} \mathcal{C} .
$$

It is the type of complexification that takes the sphere $S^{2}$ to the $\mathbf{H}_{+}^{2}$ hyperboloid . Correspondingly, the properly altered scaling transformation in eq. (11), together with $b \rightarrow i b$, will take the free motion on $S^{2}$ to the one perturbed by $\cot \theta$, the trigonometric counterpart of $\operatorname{coth} \eta$. The $\cot \theta$ potential on $S^{2}$ is exactly solvable [9] according to,

$$
\begin{aligned}
{\left[\mathbf{L}^{2}-2 b \cot \theta\right] \mathcal{X}_{t}^{\widetilde{m}}(\theta, \varphi) } & =\left[\widetilde{\mathbf{L}^{2}}-\frac{\alpha_{t}^{2}}{4}\right] \mathcal{X}_{t}^{\widetilde{m}}(\theta, \varphi)=\left[t(t+1)-\frac{\alpha_{t}^{2}}{4}\right] \mathcal{X}_{t}^{\widetilde{m}}(\theta, \varphi), \\
\widetilde{\mathbf{L}^{2}} & =e^{-\frac{\alpha_{t} \theta}{2}} B_{t}(\varphi) \mathcal{C} e^{\frac{\alpha_{t} \theta}{2}} B_{t}^{-1}(\varphi), \quad \alpha_{t}=\frac{2 b}{t+\frac{1}{2}}
\end{aligned}
$$

The $B_{t}(\varphi)$ matrices are the trigonometric analogues to $A_{t}(\varphi)$ in (111) and (12). Also in this case, the wave functions of the perturbed motion continue transforming according to so(3) irreps but now represented by $\mathcal{X}_{t}^{\widetilde{m}}(\theta, \varphi)$, the eigenfunctions of the Casimir invariant, $\widetilde{\mathbf{L}}^{2}$, of the non-unitarily transformed so(3) algebra,

$$
\mathcal{X}_{t}^{\widetilde{m}}(\theta, \varphi)=\mathcal{F}_{t}^{|\widetilde{m}|}(\theta) e^{i \widetilde{m} \varphi} \quad t=0,1,2, . ., \quad|\widetilde{m}| \in[0, t],
$$

where

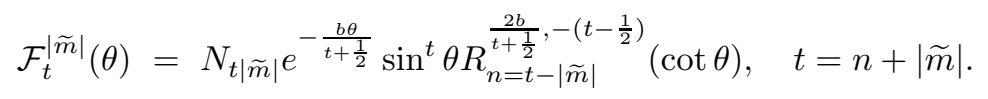

The $B_{t}$ matrices in (15) in first instance describe the decomposition of the non-exponential part of $\mathcal{F}_{t}^{|\widetilde{m}|}(\theta)$ into associated Legendre functions, $P_{t}^{|m|}(\theta)$, with $|m| \in[|\widetilde{m}|, t]$. Explicit expressions are given in [9]. Though the $t$-label in the last equation no longer has the meaning of ordinary angular momentum, it nonetheless continues labeling the $s o(3)$ irreps in the new (potential) representation of the algebra, and $\widetilde{m}$, continues obeying same branching rule with respect to $t$ as does the ordinary magnetic quantum number $m$ with respect to conventional orbital angular momentum, $l$. However, the Euclidean distance, $r^{2}=x^{2}+y^{2}+z^{2}$, is no longer conserved by transformations generated by the algebra elements in the new representation. Rather, one encounters the deformed metric,

$$
\left(x^{2}+y^{2}+z^{2}\right) \longrightarrow e^{-2 b \theta}\left(x^{2}+y^{2}+z^{2}\right) .
$$




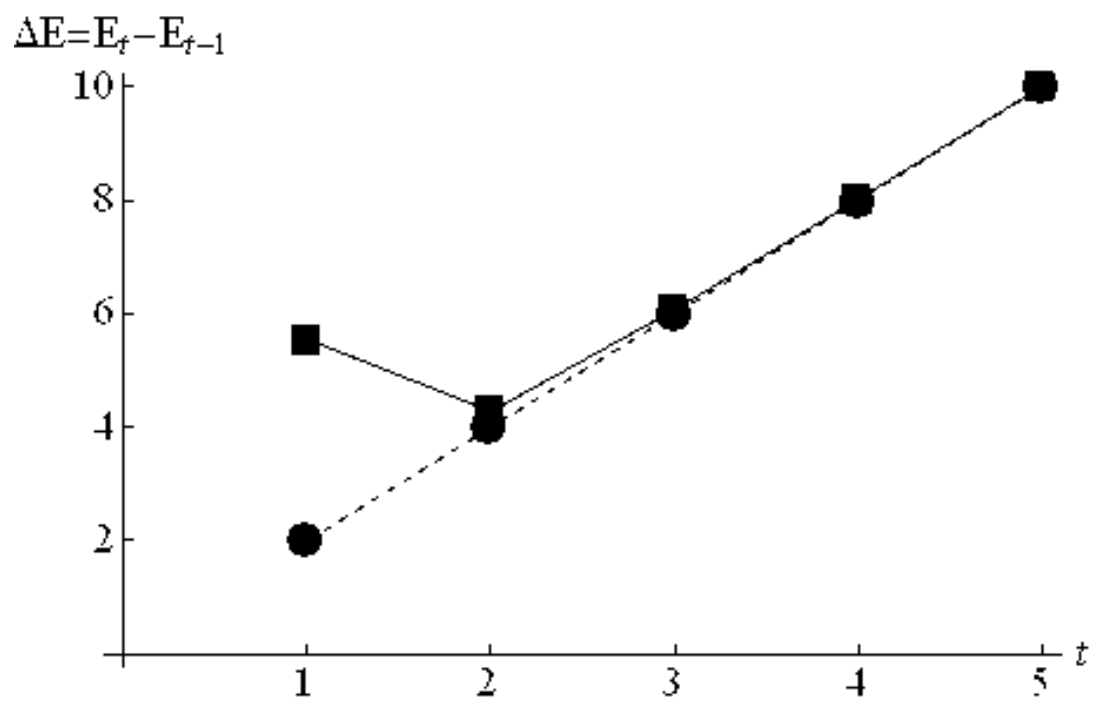

FIG. 2: The splitting, $\Delta E=\left(E_{t}-E_{t-1}\right)$, for a moderate $b=1$ value, between neighboring rotational levels belonging to the ordinary rigid rotator (circles connected by a dashed line) and the cotangent-perturbed rigid rotator (squares connected by a solid line). The rotational bands of the perturbed rotator show a typical anomalous splitting between the ground state and its first excitation. For the higher lying excitations the perturbed level splittings rapidly approach the unperturbed ones, a behavior visualized by practically coincident squares and circles.

The representation functions $\mathcal{X}_{t}^{\tilde{m}}(\theta, \varphi)$ of the non-unitarily transformed $s o(3)$ in eqs. (16), (17) are defined in terms of non-classical Romanovski polynomials [10], $R_{n}^{\alpha, \beta}(\cot \theta)$, which satisfy the following hyper geometric differential equation,

$$
\left(1+x^{2}\right) \frac{\mathrm{d}^{2} R_{n}^{\alpha, \beta}}{\mathrm{d} x^{2}}+2\left(\frac{\alpha}{2}+\beta x\right) \frac{\mathrm{d} R_{n}^{\alpha, \beta}}{\mathrm{d} x}-n(2 \beta+n-1) R_{n}^{\alpha, \beta}=0,
$$

and are obtained from the weight function

$$
\omega^{\alpha, \beta}(x)=\left(1+x^{2}\right)^{\beta-1} \exp \left(-\alpha \cot ^{-1} x\right),
$$

by means of the Rodrigues formula:

$$
R_{n}^{\alpha, \beta}(x)=\frac{1}{\omega^{\alpha, \beta}(x)} \frac{\mathrm{d}^{n}}{\mathrm{~d} x^{n}}\left[\left(1+x^{2}\right)^{n} \omega^{\alpha, \beta}(x)\right] .
$$

The parameters $\alpha$ and $\beta$ (labeled by the index $t$ in the text) are

$$
\alpha=\frac{2 b}{t+\frac{1}{2}}, \quad \beta=-\left(t+\frac{1}{2}\right)+1, \quad t=n+|\widetilde{m}| .
$$

In ref. [9] it has been shown that, due to the presence of the representation constant $\alpha_{t}$ in the expression for the energy in (15), the cotangent perturbed rigid rotator describes rotational bands of anomalously large gap between the ground and the first excited sates, as illustrated in Fig. 2. In recapitulation of the above examples, it must have become clear that the potential representation of an algebra can be given the meaning of metric deformation of the initial group symmetry space, and thereby of a group symmetry breaking by the mass scale introduced by the potential strength. In the subsequent section we shall use the potential algebra concept as a guidance in the search for a potential of a conformal symmetry algebra which we then will employ as a quark potential with the aim to explain the observed conformal degeneracies in the unflavored hadron spectra.

\section{CONFORMAL SYMMETRY IN QCD AND DEGENERACIES IN HADRON SPECTRA}

The unflavored baryon spectra reported so far reveal typical conformal degeneracies, as visible from the fact that the respective nucleon $(N)$, and $\Delta(1232)$-excitations with masses between $1250 \mathrm{MeV}$ and $2500 \mathrm{MeV}$ distribute each 

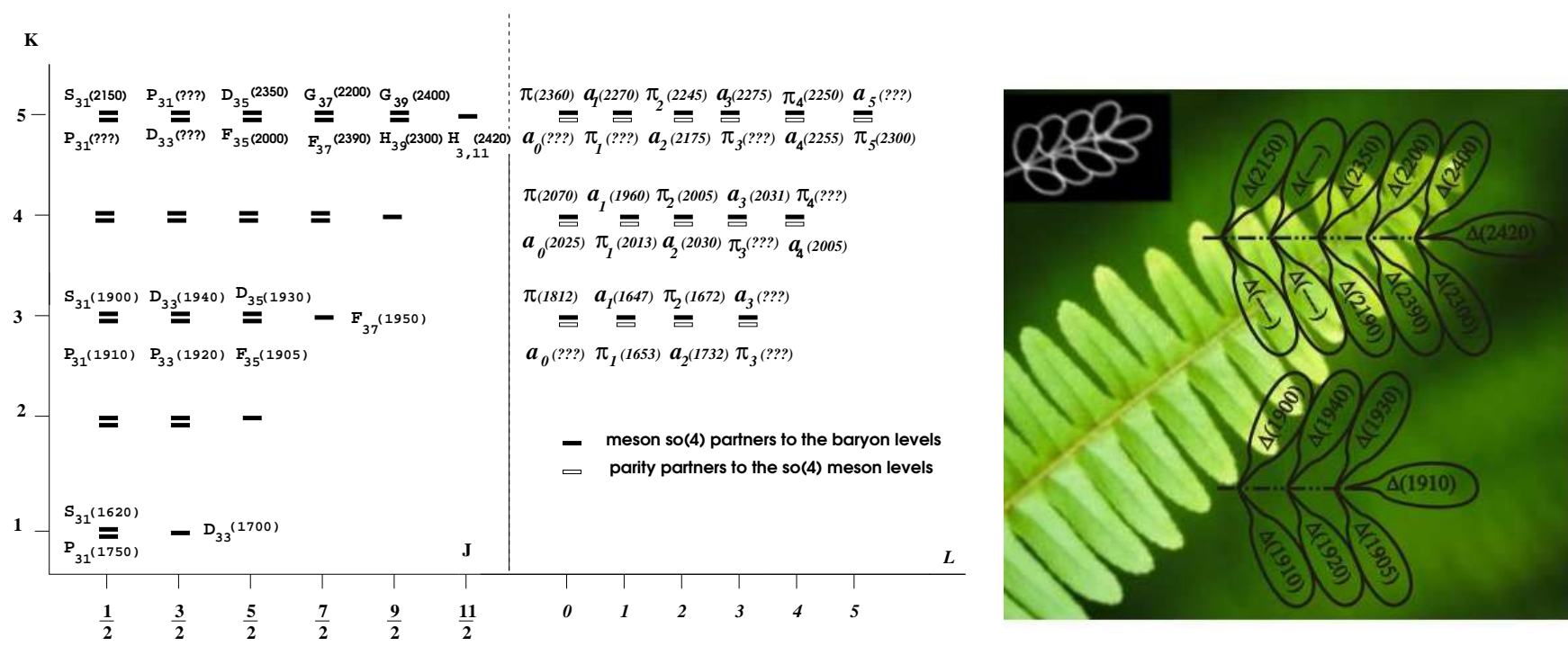

FIG. 3: $\Delta(1232)$ spectrum on the plane spin $(\mathrm{J})$, versus $4 \mathrm{D}$ angular momentum, $K$ (replaced by $(K+1)$ ) together with excited isovector mesons according to the Crystal Barrel data, compiled in [18]. Each baryon level has $K$ bifurcations into states of opposite parities, and contains a single-parity state of the maximal spin $J=(K+1 / 2)$. In resemblance of fern, we choose to term such multi-spin-parity clusters as spin- $(\mathrm{K}+1 / 2)$ ferneons (schematically represented in the right figure, courtesy $\mathrm{C}$. Compean). The gap of $\sim 500 \mathrm{MeV}$ between $\Delta(1232)$ and the spin- $3 / 2$ ferneon is significantly larger than the subsequent gaps of $\approx 200 \mathrm{MeV}$ among ferneons whose spins differ by two units, a behavior which hints on the necessity of introducing on $S^{3}$ a potential of an $s o(4)$ symmetry algebra, in parallel to the lower dimensional $S^{2}$ case in Fig. 2 . The spin-5/2 $2^{+}$and $9 / 2^{+}$ferneons are "missing". The figure is suggestive of a fermion-boson supersymmetry in the spirit of [19]. The $N$ spectrum follows similar patterns (see [11] for details).

over three so(4) levels of the type, $(K / 2, K / 2) \otimes[(1 / 2,0) \oplus(0,1 / 2)]$ with $\mathrm{K}=1,3$, and 5 [1] . Levels with $\mathrm{K}=2,4$ are "missing". Such level sets can be viewed as pieces of infinite dimensional unitary so $(2,4)$ irreducible representations and are indicative of the relevance of conformal symmetry in QCD [22]. The QCD is conformal in two regimes, in the ultraviolet (UV) where the partons are practically mass-less and the strong coupling tends to zero, and in the infrared (IR) where according to recent measurements [12], the strong coupling walks toward a fixed value (so called "conformal window"). At the mass scale of interest, the $u$ and $d$ quark masses can be viewed as small and one may consider the possibility that the aforementioned degeneracies represent a print left by the conformal symmetry. The conformal symmetry of QCD is furthermore supported by the gauge/gravity duality [13] which identifies the conformal gauge theory at the boundary of the $A d S_{5}$ space-time with QCD at high-temperatures. The $A d S_{5}$ geometry is $S^{1} \otimes S^{3}$ and the isometry algebra of $S^{3}$ is so(4), a reason for which the excitation modes of the free motion on $S^{3}$ are classified according to the so(4) irreps, and the energies are $(K+1)^{2}$-fold degenerate. This is so because free motion on $S^{3}$ describes $4 \mathrm{D}$ rotational bands in terms of the eigenvalue problem of the squared $4 \mathrm{D}$ angular momentum, $\mathcal{K}$, according to $\mathcal{K} Y_{K l m}(\chi, \theta, \varphi)=\epsilon_{K} Y_{K l m}(\chi, \theta, \varphi)$, with $\epsilon_{K}=K(K+2)$, and $Y_{K l m}(\chi, \theta, \varphi)$ standing for the 4D hyper spherical harmonics. Here, $\chi$, and $\theta$ denote the two polar angles, while $\varphi$ is the azimuthal angle. The $S^{3}$ metric is given by $d s^{2}=d \chi^{2}+\sin ^{2} \theta\left(d \theta+\sin ^{2} \varphi d \varphi\right)$. Comparison of these $4 \mathrm{D}$ rotational bands with data on the $N$ and $\Delta$ excitations reveals a good match between the quantum numbers, but the respective bands are characterized by anomalous gaps between the ground state and the first excited state, as visible from Fig. 3. In order to describe this observation we approximate, for the time being, the hadrons by two-body systems, $q-(q q)$ for baryons, and $q-\bar{q}$ for mesons, and replace them afterward by scalar particles on $S^{3}$ of the corresponding reduced mass, which we treat as free parameters. On the basis of the experience made in the previous section it is not difficult to realize that it is the cotangent-perturbed $4 \mathrm{D}$ rigid rotator on $S^{3}$ that can be expected to generate $4 \mathrm{D}$ rotational bands with the required anomalous gap between the ground state and its first excitation mode. The $\cot \chi$ potential, first introduced by Schrödinger [14] as a harmonic function on $S^{3}$, derives its utility for employment in the description of hadron spectra from its close relationship to the Cornell potential, predicted by lattice QCD, as visible from its Taylor series decomposition [15],

$$
-2 b \cot \frac{\widehat{r}}{R}=-\frac{2 b R}{\widehat{r}}+\frac{4 b}{3 R} \widehat{r}+\ldots, \quad \text { for } \quad \chi=\frac{\widehat{r}}{R}
$$


where $\widehat{r}$ denotes the geodesic distance on $S^{3}$, and $R$ is the $S^{3}$ radius. Conformal symmetry implies $R$ independence. The $s o(4)$ symmetry algebra of this potential has been explicitly constructed in [16] following the scheme explained in the previous section, namely, establishing the equivalence

$$
\begin{array}{r}
(\mathcal{K}-2 b \cot \chi) \Psi_{K \tilde{l} \widetilde{m}}(\chi, \theta, \varphi)=\left(\widetilde{\mathcal{K}}-\frac{\alpha_{K}^{2}}{4}\right) \Psi_{K \tilde{l} \tilde{m}}(\chi, \theta, \varphi)=\left(\left(K(K+2)-\frac{\alpha_{K}^{2}}{4}\right) \Psi_{K \tilde{l} \tilde{m}}(\chi, \theta, \varphi),\right. \\
\Psi_{K \widetilde{l} \tilde{m}}(\chi, \theta, \varphi)=e^{\frac{\alpha_{K} \chi}{2}} \psi_{K}^{\widetilde{l}}(\chi) Y_{\widetilde{l}}^{\widetilde{m}}(\theta, \varphi), \quad \psi_{K}^{\widetilde{l}}(\chi)=\sin ^{K} \chi R_{K-\widetilde{l}}^{\alpha_{K}, \beta_{K}-1}(\cot \chi), \quad \alpha_{K}=-\frac{2 b}{K+1}, \quad \beta_{K}=-K,
\end{array}
$$

with

$$
\widetilde{\mathcal{K}}=e^{\frac{\alpha_{K} \chi}{2}} \mathbf{A}_{K} \mathcal{K} e^{-\frac{\alpha_{K} \chi}{2}} \mathbf{A}_{K}^{-1}
$$

The matrices $\mathbf{A}_{K}$ realize in first instance the expansions of the $\psi_{K}^{\widetilde{l}}(\chi)$ functions into $\sin ^{l} \chi \mathcal{G}_{K-l}^{l+1}(\cos \chi)$, with $l \in[\widetilde{l}, K]$, and $\mathcal{G}$ being the Gegenbauer polynomials. The eigenvalue problem of the Casimir invariant $\widetilde{\mathcal{K}}$ of the non-unitarily transformed so(4) algebra is equivalent to the cot $\chi$ perturbed rigid rotator on $S^{3}$, the symmetry space of the orthogonal group $S O(4)$. The line element on the exponentially rescaled $S^{3}$ has been found as [17],

$$
\mathrm{d}^{2} \widetilde{s}=e^{-b \chi}\left(\left(1+b^{2} / 4\right) \mathrm{d}^{2} \chi+\sin ^{2} \chi\left(\mathrm{d} \theta^{2}+\sin ^{2} \theta \mathrm{d}^{2} \varphi\right)\right) .
$$

The latter equation shows that the energy scale introduced by the potential strength provides the deformation of the $S O(4)$ symmetry space. As long as $S O(4) \subset S O(2,4)$, also the conformal group symmetry will be broken by same scale. On the other side, conformal symmetry is broken by the dilaton mass and it is worth fitting the potential strength to data with the aim to see how the potential strength compares to the dilaton mass. In [17] such an analysis has been performed on the basis of the data on the high-lying unflavored meson excitations, in the compilation of [20]. We here show in Fig. 4 the data on the isotriplet vector meson excitations for illustrative purposes. The rest of the data can be consulted in [17. The data on the spectra of the high-lying unflavored mesons show not only pronounced conformal grouping patterns above $\approx 1250 \mathrm{MeV}$ but also the fermion-meson degeneracies shown in Fig. 3. Our case is that these patterns emerge in consequence of the breaking of the $S O(2,4)$ group symmetry at the level of the representation of its algebra as made evident through eq. (26), and conjecture that such a breaking may be attributed to the dilaton. In [17] a $b$-value of $b=3.2793 \pm 0.0697$ has been obtained, which is to a very good approximation isospin independent, a circumstances that speaks in favor of a possible universality. Also the $S^{3}$ radius, $R$ has been treated as a free parameter, with the aim to obtain its inverse, $1 / R$, which defines the finite temperature. In units of mass, one finds, $\hbar c \sqrt{b} / R=673.7 \mathrm{MeV}$, which hits the size of the light dilaton mass predicted in [21]. Also the value calculated for the temperature, $\mathrm{T}=\hbar c / R=373 \mathrm{MeV}$, seems reasonable insofar as it is significantly, though not much larger, than $\Lambda_{Q C D} \approx 175 \mathrm{MeV}$. Our conclusion is that the conformal symmetry in the unflavored sector in QCD may throughout be broken by the dilaton mass in the subtle way at the level of the group and conserved as potential algebra of the interaction, thus preserving the conformal degeneracies. The observed and described conformal grouping patterns in the 1250-2500 MeV excitation region of the unflavored hadrons, both baryons [22] and mesons, are in our opinion representative at least for the realization of a partial conformal dynamical symmetry in the spirit of [23]. Our findings are suggestive of a generalization of the traditional algebraic Hamiltonians [24] from $H=\sum_{r} \mathcal{C}^{r}$ to $\mathcal{H}=\sum_{p}\left(\mathbf{F} C \mathbf{F}^{-1}+\gamma_{\ell}\right)^{p}$, for suited non-unitary $\mathbf{F}$ 's, and properly introduced representation constants, $\gamma_{\ell}$ where $\ell$ is a generic representation label. Such a scheme could be helpful in improving the description of the wave functions of a variety of systems by replacing the representation functions of $S O(n) / S O(p, q)$ groups by more adequate expressions.

\section{Acknowledgments}

The efforts of the organizers in making possible the memorable event Beauty in Physics:Theory and Experiment, May 14-18, 2012 at Cocoyoc, Mexcio, where this talk was given to honor the 70th birthday of Francesco Yachello and his trailblazing work, are highly appreciated. Special thanks to David Edwin Alvarez Castillo, Cliffor Benjamin Compean Jasso, Nehemias Leija Martinez, Adrian Pallares Rivera, Alfredo Raya, Alvaro Peréz Raposo, and Hans Jürgen Weber for invaluable collaboration and support over the last eight years. I am furthermore indebted to Jose Limon Castillo for permanent assistance in computer matters.

[1] G. A. Natanzon G., Theor. Math. Phys. 38, 219 (1979). 


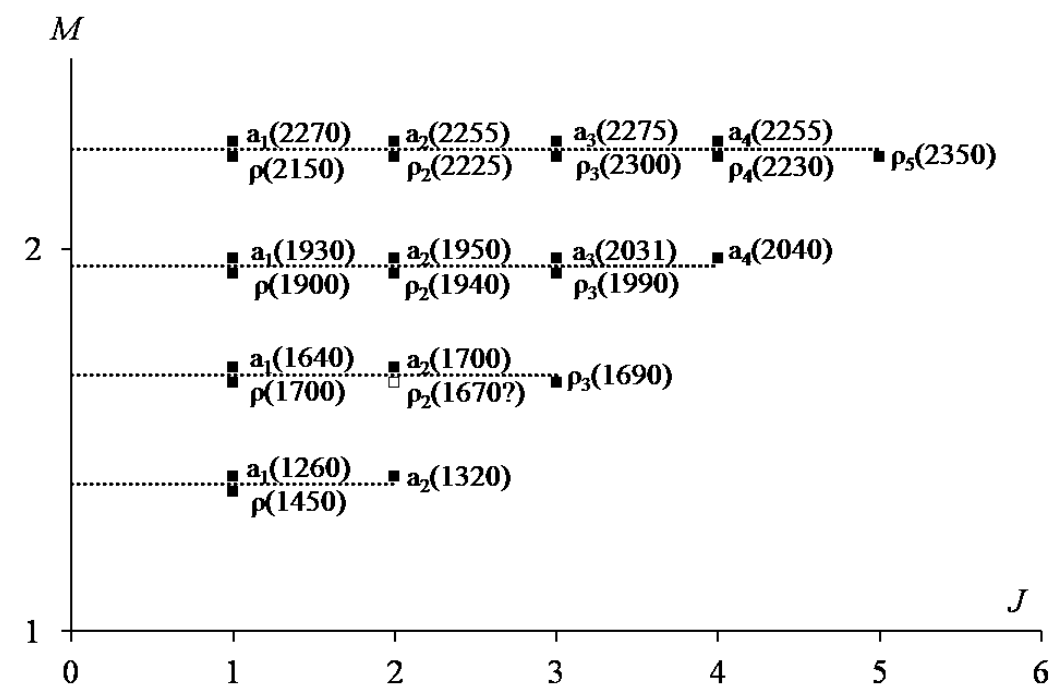

FIG. 4: High-lying excitations of the isotriplet vector mesons according to the compilation of [20] which places mesons of opposite $G$ and $C$ parities in same so(4) level. Remarkable, the narrow parity doubling of the levels, likely to be indicative of the chiral symmetry restoration from the spontaneously broken Goldstone- to the manifest Wigner mode in this excitation region.

[2] C. Rasinariu, J. V. Mallow, and A. Gangopadhyaya, C. Eur. J. Phys. 5, 111 (2007).

[3] Y. Alhassid, F. Gürsey, and F. Yachello. Phys. Rev. Lett. 50, 873 (1983).

[4] M. J. Englefield and C. Quesne, J. Phys. A:Math. Gen. 24, 3357 (1991).

[5] E. G. Kalnins, W. Miller, Jr., and G. S. Pogosyan, Phys. At. Nucl. 65, 1086 (2002).

[6] M. F. Manning and N. Rosen, Phys. Rev. 44, 951 (1933).

[7] N. Leija-Martinez, D. E. Alvarez-Castillo, and M. Kirchbach, Symm.Int.Geom.:Meth.Appl.(SIGMA) 7, 113 (2011).

[8] J. Wu and Y. Alhassid, J. Math. Phys. 31, 557 (1990).

[9] D. E. Alvarez-Castillo, C. B. Compean, and M. Kirchbach, Mol. Phys. 109, 1477 (2011).

[10] A. Raposo, H.-J. Weber, D. E. Alvarez-Castillo, and M. Kirchbach, C. Eur. J. Phys. 5, 253 (2007).

[11] M. Kirchbach, M. Moshinsky, and Yu. F. Smirnov, Phys. Rev. D 64, 114005 (2001).

[12] A. Deur, V. Burker, J. P. Chen, and W. Korsch, Phys. Lett. 665, 349 (2008).

[13] J. Maldacena, Phys. Rev. Lett. 80, 4859 (1998).

[14] E. Schrödinger, Proc. Roy. Irish Acad. 46, 183 (1941).

[15] C. Compean and M. Kirchbach, Eur. J. Phys. A 33 , 1 (2007).

[16] A. Pallares-Rivera and M. Kirchbach, J. Phys. A:Math.Theor. 44, 44530 (2011).

[17] M. Kirchbach, A. Pallares-Rivera, C. Compean, and A. Raya, arXiv:1202.0545[hep-ph]

[18] S. S. Afonin, Eur. Phys. J. A 29, 327 (2006).

[19] F. Iachello and P. Van Isacker, The Interacting Boson Model (Cambridge Univ. Press, Cambridge, 1987).

[20] S. S. Afonin, Int. J. Mod. Phys. A 23, 4205 (2008).

[21] B.-Y. Park, M. Rho, and V. Vento, Nucl. Phys. A 807, 28 (2008).

[22] M. Kirchbach and C. Compean, Phys. Rev. D 82, 034008 (2010).

[23] J. E. Garcia-Ramos, A. Leviatan, and P. Van Isacker, Phys. Rev. Lett. 102, 112502 (2009).

[24] F. Iachello, N. C. Mukhopadhyay, and L. Zhang, Phys. Rev. D 44, 898 (1991). 